\title{
Cytokine profiles in pediatric multiple sclerosis
}

\author{
Vikram Bhise ${ }^{1,4^{*}}$, Konstantin Balashov ${ }^{1}$, Marc Sturgill ${ }^{2}$, Lauren Krupp ${ }^{3}$ and Suhayl Dhib-Jalbut ${ }^{1}$
}

\begin{abstract}
Background: The immunopathogenesis of pediatric multiple sclerosis (MS) is not well understood.

Methods: We studied the cytokine profile in pre-treatment serum specimens of 19 pediatric MS patients, 25 adult MS patients, and 22 age- and gender-matched pediatric healthy controls. In addition to IL-2, IL-12p40, IL-12p70, IL-18, IL-23, IL-6, TNF-a, TGF- $\beta-1$, IFN- $\gamma$, IL-17A, IL-21, IL-10, IL-4, IL-5, IL-13, and GM-CSF, we measured osteopontin and soluble VCAM-I.

Results: In children with MS, significantly lower levels of IL-6 were present compared to age- and gender-matched healthy control children $(p<0.05)$. Moreover, significantly higher levels of osteopontin $(p<0.02)$ and sVCAM-1 $(p<0.02)$ and lower levels of IL-6 $(p<0.01)$ were present, with trends toward lower levels of IL-12p70 $(p=0.074)$ and IL-17a $(p=$ $0.05)$ compared to adults with MS.

Conclusions: These findings indicate important differences in cytokine signatures in children with MS and suggest an unexpected possible lower inflammatory cytokine profile in children with MS.
\end{abstract}

Keywords: Multiple sclerosis, Child, Cytokines, Interleukin-6, Osteopontin, Soluble vascular cell adhesion molecule

\section{Background}

The pathophysiology of multiple sclerosis (MS) is orchestrated by an array of molecular messengers known as cytokines. These signaling molecules are produced by or modulate the activity of T-cells and other immune cells. Many studies in adult MS have examined individual cytokines or their profiles to better characterize the pathogenesis of this disease, but only a limited number of studies have examined pediatric patients with MS.

Studies in experimental autoimmune encephalomyelitis (EAE), the animal model of MS, and in adult MS patients have identified disease-associated cytokines that are thus characterized as pro-inflammatory and conversely anti-inflammatory for those associated with disease remission or amelioration. In serum and CSF studies of adult MS patients, elevations of proinflammatory IL-2, IL-12, IL-6, TNF- $\alpha$, and IFN- $\gamma$, as well as anti-inflammatory/regulatory IL-10, IL-4, and

\footnotetext{
*Correspondence: bhisevi@rwjms.rutgers.edu

${ }^{1}$ Rutgers University-Robert Wood Johnson Medical School, New Brunswick, USA

${ }^{4}$ Child Health Institute, 89 French Street, Suite 2200, New Brunswick, NJ 08901, USA

Full list of author information is available at the end of the article
}

TGF- $\beta$-1 cytokines, are identified [1]. A recent study by Martins et al of 833 adult subjects with MS and 117 healthy controls using a protocol similar to our study found elevations in pro-inflammatory IFN- $\gamma$, IL-2, IL-1B, and TNF- $\alpha$, and anti-inflammatory IL-4, IL-10, and IL13 [2]. Higher levels of IL-18, IL-23, and IL-17 are also seen in the serum of MS patients compared to healthy controls [3].

Pediatric MS has distinct features from adult MS insinuating important differences are to be found in the immune profile. The vast majority of patients (over $95 \%)$ present with a relapsing-remitting course. Compared to adults with MS, pediatric-onset individuals have higher relapse rates and shorter times to their second attack, but better recovery from relapses $[4,5]$. CSF studies in younger children possess more inflammatory features with greater neutrophilic counts than older children, and are less likely to demonstrate oligoclonal banding [6]. Additionally, for these younger patients, MRI lesions may resolve entirely over the course of a few months [7]. Disability milestones take on average 5 years longer to reach in children compared to adults 
with MS [8]. These features collectively imply a greater inflammatory pathophysiology in childhood MS.

Published studies in pediatric MS pathophysiology have focused on humoral and cellular aspects of the disease. Children with central demyelinating disease demonstrate increased peripheral T-cell proliferation responses to myelin-based and dietary antigens when compared to healthy controls [9]. Additionally, there may be elevated myelin oligodendrocyte glycoprotein (MOG) antibodies, particularly in patients under 10 years of age $[10,11]$, and higher affinity antibodies present for myelin-basic protein in children [12]. A recent study identified elevated CSF complement anaphylatoxins C5a, C4a, and C3a, and CSF IL-6 levels in children with either monophasic central demyelination or MS, higher in the former, when compared to other neurological diseases [13]. In addition, plasma MOG antibodies in monophasic disease and MS both correlated with CSF IL-6 levels. These differences confirm evidence of abnormal immune physiology in children with demyelinating disease, but lack comparison to adult cohorts.

Our specific aims in this study were to establish a baseline cytokine profile in pediatric MS patients and to compare this profile to that in age- and gender-matched healthy controls and adults with MS to identify cytokine signatures unique to pediatric-onset MS.

\section{Methods}

MS patients were enrolled in the study at the time of an outpatient clinic visit to the MS Comprehensive Care Center at RWJMS, the Child Health Institute of NJ, and the Pediatric MS Center at Stony Brook, NY and met 2010 McDonald [14] and/or International Pediatric MS Study Group (IPMSSG) consensus criteria for MS [15]. Peripheral pre-treatment serum samples were collected from 19 pediatric MS patients, 25 adult MS patients, and 22 age- and gender-matched pediatric healthy controls, centrifuged at $1500 \mathrm{rpm}$ for 15-20 minutes and then stored as serum in a $-70{ }^{\circ} \mathrm{C}$ freezer. Cytokine analysis was performed using the Cytometric Bead Array immunoassay at a commercial laboratory center (Millipore). The MILLIPLEX ${ }^{\mathrm{m}}$ MAP technology uses microspheres color coded with two fluorescent dyes each and coated with specific antibodies. Following capture, an analyte is bound by a biotinylated detection antibody and then incubated with a Streptavidin-Phycoerythrin conjugate, completing the reaction. The microspheres then pass through two lasers, the first exciting the internal dye, the second, the conjugate dye. High-speed digital processors quantify the fluorescent signals. Multiple microspheres may be used to detect multiple analytes in a single ultra low volume sample.

The following cytokines were tested: IL-2, IL-12p40, IL12p70, IL-18, IL-23, IL-6, TNF- $\alpha$, IFN- $\gamma$, IL-17A, IL-21, and osteopontin as measures of pro-inflammatory activity, and IL-10, IL-4, IL-5, IL-13, GM-CSF, sVCAM-I, and TGF- $\beta-1$ as measures of anti-inflammatory activity.

All enrolled pediatric MS patients were post-pubertal. Clinical data for pediatric MS subjects collected included most recent prior brain MRI lesion count, contrastenhancing lesion number, T1 hypointense black hole number, time from first event, time from last event, total number of prior attacks, time to next clinical attack, CSF oligoclonal bands, CSF IgG index, CSF white blood cell (WBC) count, and nearest serum vitamin D level. Patients did not receive IV steroids within the 30 days prior to the specimen collection and were naive to disease-modifying therapy. Adult MS patients had fewer overall relapses and longer disease durations on average.

Statistical analysis was performed using SigmaPlot. Results classified as below laboratory detection standards were scored as 0 . Data was analyzed for normality testing followed by application of the Mann-Whitney rank sum test, except for unpaired two-tailed T-test on sVCAM data which was normally distributed with equal variances. Statistical significance was determined by $\mathrm{p}$-value $<0.05$. Fisher's exact test and student T-test were used to compare patient demographic data. Post hoc-analyses were conducted for vitamin D level, number of attacks, CSF WBC count, time from last clinical attack to blood draw, time from first clinical attack to blood draw (i.e. disease duration), baseline MRI T2 lesion number, and presence of gadoliniumenhancing lesions on baseline MRI.

\section{Results}

Mean age and gender distribution were not significantly different between healthy controls and pediatric MS patients (see Table 1), nor gender distribution between adult and pediatric MS. Differences in several cytokines were identified.

\section{Comparison to healthy controls}

Lower levels of IL-6 were seen in patients with pediatric MS compared to pediatric healthy controls $(p=0.046)$ (see Table 2). Patients with pediatric MS also had lower levels of GM-CSF, IFN- $\gamma$, IL-12p70, and IL-17A compared to healthy controls, but these differences were not identified as significantly different. A non-significant elevation in IL-23 was also observed in pediatric MS patients compared to pediatric healthy controls $(p=0.11)$.

\section{Comparison to adult MS}

Levels of interferon (IFN)-gamma were lower in pediatric MS patients compared to both adult MS patients and healthy pediatric controls, though not significant. IL-12p70 was also lower in pediatric MS patients compared to adults with MS but not significant $(p=0.074)$. 
Table 1 Demographics

\begin{tabular}{llll}
\hline & Untreated Pediatric MS & Untreated Adult MS & Pediatric Healthy Controls \\
\hline Female & $12(63 \%)^{*}$ & $19(76 \%)^{* * *}$ & $16(73 \%)$ \\
Male & $7(37 \%)$ & $6(24 \%)$ & $6(27 \%)$ \\
Age mean (SD) & 16.0 years $(1.4)^{* *}$ & 37.9 years $(10.5)$ & 15.6 years $(1.7)$ \\
Age range $(y)$ & $13.4-18.0$ & $19.0-54.7$ & $12.3-17.9$ \\
No. attacks mean & 2.1 & $1.4^{* * * *}$ \\
Disease duration mean (months) & 6.9 & 54.5 \\
${ }^{*} p=0.74 \mathrm{HC}$ vs PMS, ${ }^{* *} p=0.33 \mathrm{HC}$ vs PMS, ${ }^{* * *} p=0.51$ HC vs AMS, ${ }^{* * * *} p=0.08$ PMS vs AMS (in past 2 years)
\end{tabular}

Similarly, levels of IL-17A and IL-6 were lower compared to adults with MS ( $p=0.050$ and $p=0.004$ respectively). Adult MS patients had lower sVCAM-1 levels than patients with pediatric MS $(p=0.015)$. However, no difference in sVCAM-1 was noted between pediatric healthy controls and patients with pediatric MS.

\section{Clinical correlation}

No significant correlations were present for patients with vs without contrast-enhancing brain MRI lesions, number of clinical attacks prior to sample collection, total CSF WBCs, or MRI total brain lesion count with serum levels of cytokines. Testing for CSF oligoclonal bands was positive in all but one patient but the number of unique bands was listed for only a few. CSF IgG index on the other hand was only available for 6 patients. Serum levels of TGF- $\beta-1$ negatively correlated $(-0.69)$ with serum vitamin D levels $(p<0.01)$. Serum IL-17F levels had a modest positive correlation (0.47) with time from first clinical attack $(p<0.05)$.

\section{Discussion}

Serum levels of IL-6 were significantly lower in children with MS compared to healthy controls $(p=0.046)$ and adults with MS $(p=0.004)$. In contrast, serum IL-6 appears to correlate positively with disease activity in other inflammatory childhood diseases such as Kawasaki disease, juvenile inflammatory arthritis (JIA), and ulcerative colitis [16-18]. Indeed, anti-IL-6 therapy is currently in use for JIA [19]. IL-6 is a multifunctional cytokine produced by many cell types in response to infection, but also plays a pro-inflammatory role in incurring EAE [20-22]. These actions are mediated by the soluble form of the IL- 6 receptor (IL-6R) that may be induced by upregulation of ICAM-1 and VCAM-1, which allows $\mathrm{T}_{\mathrm{H}} 1$ cell entry into the CNS. Previous studies have shown elevated IL-6 levels in the CSF of patients with ADEM which correlates with the presence of anti-MOG antibodies. [13, 23, 24] In MS lesions, T-cells, microglia, and activated astrocytes secrete IL- 6 in the setting of augmented receptor responses [25]. This cytokine also appears to stimulate $\mathrm{T}_{\mathrm{H}} 17$ differentiation and inhibits TGF- $\beta$ Treg cell formation [25]. IL-6 also maintains a separate anti-inflammatory and neuroprotective function through oligodendrocyte differentiation, neurotropism, and peripheral nerve regeneration mediated by the membrane-bound IL-6R on select cells such as microglia [26]. Human monocytes are directed away from differentiation into dendritic cells and toward macrophages which have increased anti-inflammatory IL-4 and IL-10 and decreased IL-1 $\beta$ production [27]. Given the dual roles of IL- 6 , it is possible the relative balance between the two pathways shifts with age. Further studies could examine prepubertal patients with MS to search for even starker differences.

Cytokine levels for IL-6 were found to be significantly lower in patients with pediatric MS than adults with MS, as were IFN- $\gamma$, IL-12p70, and IL-17A (though not statistically significant), while osteopontin and sVCAM were higher. These findings are somewhat surprising, as all the markers save for osteopontin and SVCAM that were identified as significantly different indicate a greater inflammatory

Table 2 Select mean serum cytokine values

\begin{tabular}{lllllllll}
\hline & IL-6 & SVCAM-1 & Osteopontin & IL-17A & GM-CSF & IFN- $\gamma$ & IL-12p70 & IL-23 \\
\hline Peds MS $(n=19)$ & $2.1(8)$ & $479,253(120,408)$ & $16,506(8913)$ & $19.3(28)$ & $10.2(40)$ & $41.6(64)$ & $11.9(28)$ & $1599(4809)$ \\
Adult MS $(n=24)$ & $12.5(22)$ & $408,110(118,417)$ & $10,557(5336)$ & $40.9(44)$ & $7.3(19)$ & $72.1(91)$ & $33.3(57)$ & $490(1307)$ \\
Pediatric Healthy Controls $(n=22)$ & $18.2(31)$ & $497,982(128,890)$ & $14,240^{*}$ & $41.0(54)$ & $40.1(109)$ & $74.6(91)$ & $39.6(86)$ & $820(1499)$ \\
& & & $(10,718)^{* *}$ & & & & & \\
HC vs Peds MS $(p)$ & $\mathbf{0 . 0 4 6}$ & 0.637 & 0.255 & 0.454 & 0.302 & 0.408 & 0.112 & 0.108 \\
Adult MS vs Peds MS $(p)$ & $\mathbf{0 . 0 0 4}$ & $\mathbf{0 . 0 1 5}$ & $\mathbf{0 . 0 2 2}$ & $\mathbf{0 . 0 5 0}$ & 0.742 & 0.248 & 0.074 & 0.334 \\
\hline
\end{tabular}

* Mean value of cytokine concentration in serum in $\mathrm{pg} / \mathrm{ml}$

** Standard deviation values are listed in parentheses ()

Significant findings listed in bold 
profile in adults. Given the seemingly greater inflammatory clinical nature of patients with pediatric MS, it was anticipated that that all these markers would be elevated instead relative to adults with MS. This may represent a substantial finding which challenges the little-known pathophysiology in early-onset MS. These results highlight that these particular cytokines may be useful markers of disease activity. Alternatively, given the small sample size, these findings may be driven by chance (Type 1 error), for example by inadvertent selection of patients with relatively low disease activity.

Soluble vascular cell adhesion molecule-I (sVCAM-I) is elevated in MS patients and correlates with clinical and MRI disease activity [28-30]. Five samples were found to be erroneously collected from post-treatment patients, 3 with Rebif and 2 with Copaxone all started a few months prior to sample collection. These patients were not included (original $n=24$ ) in the statistical analysis. The sample size of 5 patients may be too low to support meaningful findings, but we did note elevated mean levels of osteopontin and sVCAM-1 in the treated patients compared to untreated patients (as well as controls), which was significant for $\operatorname{sVCAM-1}(p=0.011)$. In line with prior studies, interferon- $\beta$ increases sVCAM levels [31-33], but rather is noted to downregulate osteopontin [34]. It is unclear if osteopontin represents a variant pathway in pediatric MS.

The negative correlation identified between serum levels of TGF- $\beta-1$ and serum vitamin D levels $(p<0.01)$ highlights other studies in MS and findings that vitamin D supplementation may increase TGF- $\beta$ expression, which may play a role in its mechanism of action [35-37]. TGF- $\beta-1$ is an anti-inflammatory cytokine produced by regulatory T-cells. In mouse models of EAE, vitamin D administration induces TGF- $\beta-1$ production, [38], while exogenous TGF- $\beta-1$ ameliorates the disease [39]. Human derived T-cell lines from active MS patients produce less TGF- $\beta-1$ than stable patients [40]. Other studies indicate effects on TGF- $\beta$-2 instead [41]. In our pretreatment cohort, we may be seeing compensatory efforts to elevate TGF- $\beta$ where vitamin $D$ is lacking.

The correlation between serum IL-17 F levels and time from first clinical attack $(p<0.05)$ may explain the lack of certain significant findings in cytokine profiles if time from clinical attack is relevant. Thus, acquiring samples at the time of relapse may be informative as well and a useful goal for future studies. Lower levels of IL-17 at the time of attack and lower levels of IL- 6 in children compared to adults may implicate pediatric MS as a more $\mathrm{T}_{\mathrm{H}} 1$ mediated disease. Possibly $\mathrm{T}_{\mathrm{H}} 17$ activity becomes a more prominent feature with disease chronicity or is simply not well developed in early MS. Nevertheless, IL-17 responses do appear to play a role, but may explain why concomitant responses in IL- 6 and TGF- $\beta$ are not seen [42].
Osteopontin is classified as a $\mathrm{T}_{\mathrm{H}} 1$ cytokine, as it has diverse effects on inflammation, cell survival, cell-mediated immunity, bone mineralization, and more [43, 44]. Various cells produce this cytokine which induces IL-12 and IFN- $\gamma$ and inhibits IL-10 expression from monocytes. OPN-deficient EAE mice show milder disease [45]. Elevated levels are seen in CSF and plasma of MS patients, even greater at times of relapses [46, 47].

\section{Conclusions}

A panel of inflammatory markers, namely IL-6, sVCAM1, osteopontin, and IL-17A, have been found differentially expressed in serum samples of children with MS compared to pediatric healthy controls and adults with MS. The combination of findings suggests a lower inflammatory profile in children with MS compared to adult MS, contrary to clinical expectations. Future prospective studies with larger samples sizes and cohorts including prepubertal patients are needed to validate this pilot data and assess if they are prognostic of disease outcome. The absence of longitudinal samples in this study limits further interpretation. Such studies would also benefit from serial samples including post-treatment assessment. Additional investigations should also focus on cell types that express or are targeted by the cytokines identified in this study as well as broader examination of CSF.

\section{Acknowledgements}

We would like to thank Yaritza Rosario, APN-C, MSCN and Lisa Cerracchio, $\mathrm{BSN}, \mathrm{RN}, \mathrm{CCRC}$ for their kind assistance in helping conduct the study.

\section{Funding}

Supported by a grant from the New Jersey Health Foundation, Inc to Vikram Bhise, MD.

\section{Availability of data and material}

The datasets collected and/or analyzed during the current study are available from the corresponding author on reasonable request.

\section{Authors' contributions}

VB conceived the project, recruited and consented pediatric patients, performed statistical analysis, and composed the manuscript. KB recruited and consented adult MS patients, and was a major contributor in writing the manuscript. MS performed statistical analysis on the data. LK assisted with project design and recruited and consented pediatric MS patients. SDJ assisted with project design and was a major contributor in writing the manuscript. All authors have read and approved the final manuscript.

\section{Competing interests}

The authors declare that they have no competing interests.

\section{Consent for publication}

Not applicable

\section{Ethics approval and consent to participate}

This study was approved the Rutgers-RWJMS IRB. For pediatric subjects, parental consent and patient assent forms were signed prior to study entry. Adult subjects similarly signed consent forms prior to study entry. 


\section{Description}

This study reports differences found in serum cytokine signatures in pretreatment children with multiple sclerosis (MS) compared to age- and gender-matched pediatric healthy controls and pretreatment adults with MS.

\section{Author details}

'Rutgers University-Robert Wood Johnson Medical School, New Brunswick, USA. ${ }^{2}$ Rutgers University-Ernest Mario School of Pharmacy, Piscataway, USA. ${ }^{3}$ New York University Langone Medical Center, New York City, USA. ${ }^{4}$ Child Health Institute, 89 French Street, Suite 2200, New Brunswick, NJ 08901, USA.

\section{Received: 9 July 2016 Accepted: 27 September 2016}

Published online: 03 November 2016

\section{References}

1. Imitola J, Chitnis T, Khoury SJ. Cytokines in multiple sclerosis: from bench to bedside. Pharmacol Ther. 2005;106(2):163-77.

2. Martins TB, Rose JW, Jaskowski TD, Wilson AR, Husebye D, Seraj HS, et al. Analysis of proinflammatory and anti-inflammatory cytokine serum concentrations in patients with multiple sclerosis by using a multiplexed immunoassay. Am J Clin Pathol. 2011;136(5):696-704

3. Chen YC, Chen SD, Miao L, Liu ZG, Li W, Zhao ZX, et al. Serum levels of interleukin (IL)-18, IL-23 and IL-17 in Chinese patients with multiple sclerosis. J Neuroimmunol. 2012;243(1-2):56-60

4. Trojano M, Liguori M, Bosco Zimatore G, Bugarini R, Avolio C, Paolicelli D, et al. Age-related disability in multiple sclerosis. Ann Neurol. 2002;51(4):475-80.

5. Gorman MP, Healy BC, Polgar-Turcsanyi M, Chitnis T. Increased relapse rate in pediatric-onset compared with adult-onset multiple sclerosis. Arch Neurol. 2009;66(1):54-9.

6. Chabas D, Ness J, Belman A, Yeh EA, Kuntz N, Gorman MP, et al. Younger children with MS have a distinct CSF inflammatory profile at disease onset. Neurology. 2010;74(5):399-405.

7. Chabas D, Castillo-Trivino T, Mowry EM, Strober JB, Glenn OA, Waubant E. Vanishing MS T2-bright lesions before puberty: a distinct MRI phenotype? Neurology. 2008;71(14):1090-3

8. Renoux C, Vukusic S, Mikaeloff Y, Edan G, Clanet M, Dubois B, et al. Natural history of multiple sclerosis with childhood onset. N Engl J Med. 2007;356(25):2603-13.

9. Banwell B, Bar-Or A, Cheung R, Kennedy J, Krupp LB, Becker DJ, et al. Abnormal T-cell reactivities in childhood inflammatory demyelinating disease and type 1 diabetes. Ann Neurol. 2008;63(1):98-111.

10. McLaughlin KA, Chitnis T, Newcombe J, Franz B, Kennedy J, McArdel S, et al. Age-dependent B cell autoimmunity to a myelin surface antigen in pediatric multiple sclerosis. J Immunol. 2009;183(6):4067-76.

11. Brilot F, Dale RC, Selter RC, Grummel V, Kalluri SR, Aslam M, et al. Antibodies to native myelin oligodendrocyte glycoprotein in children with inflammatory demyelinating central nervous system disease. Ann Neurol. 2009;66(6):833-42.

12. O'Connor KC, Lopez-Amaya C, Gagne D, Lovato L, Moore-Odom NH, Kennedy J, et al. Anti-myelin antibodies modulate clinical expression of childhood multiple sclerosis. J Neuroimmunol. 2010;223(1-2):92-9.

13. Horellou P, Wang M, Keo V, Chretien P, Serguera C, Waters $P$, et al. Increased interleukin-6 correlates with myelin oligodendrocyte glycoprotein antibodies in pediatric monophasic demyelinating diseases and multiple sclerosis. J Neuroimmunol. 2015;289:1-7.

14. Polman $\mathrm{CH}$, Reingold SC, Banwell B, Clanet M, Cohen JA, Filippi M, et al. Diagnostic criteria for multiple sclerosis: 2010 revisions to the McDonald criteria. Ann Neurol. 2011;69(2):292-302

15. Krupp LB, Tardieu M, Amato MP, Banwell B, Chitnis T, Dale RC, et al. International Pediatric Multiple Sclerosis Study Group criteria for pediatric multiple sclerosis and immune-mediated central nervous system demyelinating disorders: revisions to the 2007 definitions. Mult Scler. 2013;19(10):1261-7.

16. de Benedetti F, Massa M, Robbioni P, Ravelli A, Burgio GR, Martini A. Correlation of serum interleukin- 6 levels with joint involvement and thrombocytosis in systemic juvenile rheumatoid arthritis. Arthritis Rheum. 1991;34(9):1158-63.

17. Tan Z, Yuan Y, Chen S, Chen Y, Chen TX. Plasma Endothelial Microparticles, TNF-a and IL-6 in Kawasaki Disease. Indian Pediatr. 2013;50(5):501-3.

18. Wine E, Mack DR, Hyams J, Otley AR, Markowitz J, Crandall WW, et al. Interleukin-6 is associated with steroid resistance and reflects disease activity in severe pediatric ulcerative colitis. J Crohns Colitis. 2013;7(11):916-22.
19. Frampton JE. Tocilizumab: a review of its use in the treatment of juvenile idiopathic arthritis. Paediatr Drugs. 2013;15(6):515-31.

20. Eugster HP, Frei K, Kopf M, Lassmann H, Fontana A. IL-6-deficient mice resist myelin oligodendrocyte glycoprotein-induced autoimmune encephalomyelitis. Eur J Immunol. 1998;28(7):2178-87.

21. Gijbels K, Brocke S, Abrams JS, Steinman L. Administration of neutralizing antibodies to interleukin-6 (IL-6) reduces experimental autoimmune encephalomyelitis and is associated with elevated levels of IL-6 bioactivity in central nervous system and circulation. Mol Med. 1995;1(7):795-805.

22. Diab A, Zhu J, Xiao BG, Mustafa M, Link H. High IL-6 and low IL-10 in the central nervous system are associated with protracted relapsing EAE in DA rats. J Neuropathol Exp Neurol. 1997;56(6):641-50.

23. Dale RC, Morovat A. Interleukin-6 and oligoclonal lgG synthesis in children with acute disseminated encephalomyelitis. Neuropediatrics. 2003;34(3):141-5.

24. Ishizu T, Minohara M, Ichiyama T, Kira R, Tanaka M, Osoegawa M, et al. CSF cytokine and chemokine profiles in acute disseminated encephalomyelitis. J Neuroimmunol. 2006:175(1-2):52-8.

25. Janssens K, Slaets $H$, Hellings N. Immunomodulatory properties of the IL-6 cytokine family in multiple sclerosis. Ann N Y Acad Sci. 2015;1351:52-60.

26. Rothaug $M$, Becker-Pauly $C$, Rose-John S. The role of interleukin- 6 signaling in nervous tissue. Biochim Biophys Acta. 2016;1863(6 Pt A):1218-27.

27. Frisdal E, Lesnik P, Olivier M, Robillard P, Chapman MJ, Huby T, et al. Interleukin-6 protects human macrophages from cellular cholesterol accumulation and attenuates the proinflammatory response. J Biol Chem. 2011;286(35):30926-36.

28. Calabresi PA, Tranquill LR, Dambrosia JM, Stone LA, Maloni H, Bash CN, et al. Increases in soluble VCAM-1 correlate with a decrease in MRI lesions in multiple sclerosis treated with interferon beta-1b. Ann Neurol. 1997;41(5):669-74.

29. Rieckmann P, Altenhofen B, Riegel A, Baudewig J, Felgenhauer K. Soluble adhesion molecules (SVCAM-1 and sICAM-1) in cerebrospinal fluid and serum correlate with MRI activity in multiple sclerosis. Ann Neurol. 1997;41(3):326-33

30. Matsuda M, Tsukada N, Miyagi K, Yanagisawa N. Increased levels of soluble vascular cell adhesion molecule-1 (VCAM-1) in the cerebrospinal fluid and sera of patients with multiple sclerosis and human T lymphotropic virus type-1-associated myelopathy. J Neuroimmunol. 1995;59(1-2):35-40.

31. Rieckmann P, Kruse N, Nagelkerken L, Beckmann K, Miller D, Polman C, et al. Soluble vascular cell adhesion molecule (VCAM) is associated with treatment effects of interferon beta- $1 \mathrm{~b}$ in patients with secondary progressive multiple sclerosis. J Neurol. 2005;252(5):526-33.

32. Graber J, Zhan M, Ford D, Kursch F, Francis G, Bever C, et al. Interferon-beta1a induces increases in vascular cell adhesion molecule: implications for its mode of action in multiple sclerosis. J Neuroimmunol. 2005;161(1-2):169-76.

33. Bitsch A, Bahner D, Wachter C, Elitok E, Bogumil T, Dressel A, et al. Interferon beta-1b modulates serum SVCAM-1 levels in primary progressive multiple sclerosis. Acta Neurol Scand. 2004:110(6):386-92.

34. Hong J, Hutton GJ. Regulatory effects of interferon-beta on osteopontin and interleukin-17 expression in multiple sclerosis. J Interferon Cytokine Res. 2010;30(10):751-7

35. Mahon BD, Gordon SA, Cruz J, Cosman F, Cantorna MT. Cytokine profile in patients with multiple sclerosis following vitamin D supplementation. J Neuroimmunol. 2003:134(1-2):128-32.

36. Mosayebi G, Ghazavi A, Ghasami K, Jand Y, Kokhaei P. Therapeutic effect of vitamin D3 in multiple sclerosis patients. Immunol Invest. 2011:40(6):627-39.

37. Mann EH, Chambers ES, Chen YH, Richards DF, Hawrylowicz CM. 1alpha,25dihydroxyvitamin D3 acts via transforming growth factor-beta to upregulate expression of immunosuppressive CD73 on human CD4+ Foxp3- T cells. Immunology. 2015;146(3):423-31.

38. Cantorna MT, Woodward WD, Hayes CE, DeLuca HF. 1,25-dihydroxyvitamin D3 is a positive regulator for the two anti-encephalitogenic cytokines TGFbeta 1 and IL-4. J Immunol. 1998:160(11):5314-9.

39. Racke MK, Cannella B, Albert P, Sporn M, Raine CS, McFarlin DE. Evidence of endogenous regulatory function of transforming growth factor-beta 1 in experimental allergic encephalomyelitis. Int Immunol. 1992;4(5):615-20.

40. Mokhtarian F, Shi Y, Shirazian D, Morgante L, Miller A, Grob D. Defective production of anti-inflammatory cytokine, TGF-beta by T cell lines of patients with active multiple sclerosis. J Immunol. 1994;152(12):6003-10.

41. Shirvani-Farsani Z, Behmanesh M, Mohammadi SM, Naser MA. Vitamin D levels in multiple sclerosis patients: Association with TGF-beta2, TGF-betaRl, and TGF-betaRII expression. Life Sci. 2015;134:63-7. 
42. Vargas-Lowy D, Kivisakk P, Gandhi R, Raddassi K, Soltany P, Gorman MP, et al. Increased Th17 response to myelin peptides in pediatric MS. Clin Immunol. 2013;146(3):176-84.

43. O'Regan A, Berman JS. Osteopontin: a key cytokine in cell-mediated and granulomatous inflammation. Int J Exp Pathol. 2000;81(6):373-90.

44. Ashkar S, Weber GF, Panoutsakopoulou V, Sanchirico ME, Jansson M, Zawaideh S, et al. Eta-1 (osteopontin): an early component of type-1 (cell-mediated) immunity. Science. 2000;287(5454):860-4.

45. Jansson M, Panoutsakopoulou V, Baker J, Klein L, Cantor H. Cutting edge: Attenuated experimental autoimmune encephalomyelitis in eta-1/ osteopontin-deficient mice. J Immunol. 2002;168(5):2096-9.

46. Braitch M, Nunan R, Niepel G, Edwards LJ, Constantinescu CS. Increased osteopontin levels in the cerebrospinal fluid of patients with multiple sclerosis. Arch Neurol. 2008;65(5):633-5.

47. Comabella M, Pericot I, Goertsches R, Nos C, Castillo M, Blas Navarro J, et al. Plasma osteopontin levels in multiple sclerosis. J Neuroimmunol. 2005:158(1-2):231-9.

Submit your next manuscript to BioMed Central and we will help you at every step:

- We accept pre-submission inquiries

- Our selector tool helps you to find the most relevant journal

- We provide round the clock customer support

- Convenient online submission

- Thorough peer review

- Inclusion in PubMed and all major indexing services

- Maximum visibility for your research

Submit your manuscript at www.biomedcentral.com/submit 